Robin M. Turner, Katy J.L. Bell, Rachael L. Morton, Andrew Hayen, Kirsten Howard, Bruce Armstrong, John F. Thompson, and Les Irwig, School of Public Health, The University of Sydney; John F. Thompson, Melanoma Institute Australia, Sydney, New South Wales, Australia; and Anne Brecht Francken, Isala Klinieken, Zwolle, the Netherlands.

Submitted December 16, 2010; accepted June 16, 2011; published online ahead of print at www.jco.org on November 7, 2011

Supported by Grants No. 402764 and 633003 from the Australian National Health and Medical Research Council.

Authors' disclosures of potential conflicts of interest and author contributions are found at the end of this article.

Corresponding author: Robin M. Turner, Edward Ford Building (A27), School of Public Health, The University of Sydney, New South Wales 2006, Australia; e-mail: robin.turner@sydney.edu.au.

C 2011 by American Society of Clinical Oncology

0732-183X/11/2935-4641/\$20.00

DOI: $10.1200 / J C O .2010 .34 .2956$

\title{
Optimizing the Frequency of Follow-Up Visits for Patients Treated for Localized Primary Cutaneous Melanoma
}

Robin M. Turner, Katy J.L. Bell, Rachael L. Morton, Andrew Hayen, Anne Brecht Francken, Kirsten Howard, Bruce Armstrong, John F. Thompson, and Les Irwig

See accompanying editorial on page 4606

$$
\begin{array}{llllllll}
\text { A } & \text { B } & \text { S } & \text { T } & \text { R } & \text { A } & \text { C } & \text { T }
\end{array}
$$

\section{Purpose}

To develop more evidence-based guidelines for the frequency of patient follow-up after treatment of localized (American Joint Committee on Cancer [AJCC] stage I or II) melanoma.

\section{Methods}

We used data from Melanoma Institute Australia on an inception cohort of 3,081 consecutive patients first diagnosed with stage I or II melanoma between January 1985 and December 2009. Kaplan-Meier curves and Cox models were used to characterize the time course and predictors for recurrence and new primaries. We modeled the delay in diagnosis of recurrence or new primary as well as the number of monitoring visits required using two monitoring schedules: first, according to 2008 Australian and New Zealand guidelines and, second, with fewer visits, especially for those at lowest risk of recurrence.

\section{Results}

For every 1,000 patients beginning follow-up, 229 developed recurrence and 61 developed new primary within 10 years. There was only a small difference in modeled delay in diagnosis (extra 44.9 and 9.6 patients per 1,000 for recurrence and new primary, respectively, with delay greater than 2 months) using a schedule that requires far fewer visits $(3,000$ fewer visits per 1,000 patients) than recommended by current guidelines. AJCC substage was the most important predictor of recurrence, whereas age and date of primary diagnosis were important predictors of developing new primary.

\section{Conclusion}

By providing less intensive monitoring, more efficient follow-up strategies are possible. Fewer visits with a more focused approach may address the needs of patients and clinicians to detect recurrent or new melanoma.

\section{J Clin Oncol 29:4641-4646. (C) 2011 by American Society of Clinical Oncology}

\section{INTRODUCTION}

The cost of regular follow-up after treatment of American Joint Committee on Cancer (AJCC) stage I or II melanoma has been estimated at between $\$ 441$ to $\$ 628$ per person per year (2004 Medicare fee reimbursement schedule) in the United States. ${ }^{1}$ On the basis of 2007 estimates, ${ }^{2}$ there are approximately 800,000 people with a past diagnosis of melanoma living in the United States; their follow-up represents an important burden to the US health care system, up to $\$ 500$ million per year. Additionally, there is a lack of evidence for the frequency and benefit of follow-up visits after treatment for cancer. ${ }^{3,4}$

Melanoma guidelines typically recommend follow-up for patients treated for localized disease (stage I or II). This recommendation is generally based on expert opinion, with monitoring schedules informed by observed recurrence rates for patients categorized into prognostic subgroups. ${ }^{5,6}$ Development of new primaries is not usually considered, although their detection is an aim of monitoring, ${ }^{5}$ nor are the benefits and harms of the proposed monitoring schedule. Given that approximately $75 \%$ of patients detect their own recurrences, ${ }^{3,7}$ and approximately 50\% detect their own second primaries, ${ }^{8}$ follow-up visits currently may be scheduled more frequently than is necessary, with possible inefficient use of health resources and an unnecessary burden on patients. Recently, a less frequent monitoring schedule was proposed ${ }^{9}$ based on an analysis of recurrences in AJCC subgroups IA, IB, IIA, IIB, and IIC in a group of patients undergoing follow-up 
at Melanoma Institute Australia (MIA; formerly the Sydney Melanoma Unit). A modification of this schedule comprises the experimental arm of a randomized trial currently under way, in which the less frequent monitoring schedule will be compared with a conventional follow-up schedule from Dutch national guidelines. ${ }^{10}$

This study aims primarily to relate the estimated delay in diagnosis of recurrence or second primary in patients with localized stage I or II cutaneous melanoma to the number of visits needed by two different monitoring schedules. Secondarily, we estimated the effect of prognostic factors on development of recurrence or second primary tumor.

\section{METHODS}

\section{Study Design and Sample}

All patients in the MIA database treated for invasive primary cutaneous melanoma who were diagnosed by excision biopsy between January 1, 1985, and December 31, 2009, and had AJCC stage I or II disease were considered for the study. Patients were excluded if they did not have MIA-reviewed pathology, were node positive (either by sentinel lymph node biopsy [SLNB] or elective lymph node dissection), had insufficient data to allow substaging of their disease, or had missing data on primary diagnosis date or last follow-up date. Ethics approval was not required for this study.

\section{Measurement of Risk Factors}

All patients had wide local excision of primary melanoma after diagnostic biopsy. ${ }^{9}$ Treatment of regional lymph nodes was categorized as SLNB, elective lymph node dissection, or observation. Breslow thickness, ulceration, mitotic rate, and Clark level of invasion (if mitotic rate was unknown) were used to classify patient primaries into AJCC substages using the 2009 updated criteria. ${ }^{11}$

\section{Follow-Up and Outcome Events}

Data were analyzed separately for development of recurrence and development of new primary (results of analysis of composite outcome, recurrence, or new primary are presented in Appendix, online only). Recurrence was defined as the first abnormality diagnosed by histopathology (including cytology) or imaging confirming the presence of metastatic disease. Diagnosis of second primary melanoma required diagnostic biopsy and histopathology. When a patient had both recurrence and second primary, we used whichever occurred first as the outcome, and the patient was censored at this time for analysis of the alternate outcome.

Follow-up visits entailed at least a medical history and clinical examination. The general follow-up schedule for patients during the study timeframe is provided in the Appendix (online only). In addition to scheduled visits, all patients could initiate visits if they were concerned they had recurrence or new primary.

\section{Statistical Analysis}

All risk factors were included in multivariable Cox proportional hazard models predicting time to recurrence and second primary. Data on diseasefree survival were considered censored for patients who were free from recurrence or new primary at last follow-up or at 10-year follow-up, whichever was earlier. Likelihood-ratio tests were used to assess significance. Proportional hazards assumptions were checked using Schoenfeld residuals. Analysis of sensitivity to competing risks was also performed.

We assessed delay in the diagnosis of recurrence or new primary and number of visits for two different monitoring schedules over 10 years of follow-up. The two schedules were as follows:

Schedule one. On the basis of 2008 Australian and New Zealand guidelines, ${ }^{5}$ schedule one involved follow-up every 6 months for 5 years, then annually for 5 years (patients with stages IA and IB disease) or every 3 months for 5 years, then annually for 5 years (stages IIA, IIB, and IIC).

Schedule two. On the basis of recommendations of a previous report ${ }^{9}$ and modification of the experimental arm of MELFO (Melanoma
Follow-up Study), ${ }^{4}$ schedule two involved follow-up annually for 10 years (stage I); every 6 months for 2 years, then annually for 8 years (stage IIA); or every 4 months for 2 years, every 6 months during year 3 , then annually for 5 years (stages IIB and IIC).

Both schedules included a 1-month follow-up visit. We modeled the theoretic number of patients experiencing a delay in diagnosis greater than 1,2 , $3,6,9$, and 12 months, as a rate per 1,000 patients starting follow-up, for two clinical scenarios. In the first scenario (only scheduled visits), we assumed any diagnosis would be made at a scheduled visit. For the second scenario (scheduled and unscheduled visits), we assumed $75 \%$ of recurrences and $50 \%$ of new primaries would be detected by the patient or partner with no delay in diagnosis, and all patients would seek clinical attention by a maximum of 3 months for recurrence and 6 months for new primaries. Bootstrap random samples were used to estimate $95 \%$ CIs and select patients who self-detected. Detailed methods and a sensitivity analysis on the proportion of self-detecting patients are presented in the Appendix (online only). For each monitoring schedule, we calculated the number of visits required per 1,000 patients starting follow-up until time of diagnosis of recurrence or new primary, time of loss to follow-up, or a period of 10 years of follow-up.

\section{RESULTS}

A total of 3,081 patients with AJCC stage I or II disease were initially included. We excluded 83 patients from analysis for whom it was not possible to obtain information on the last clinic consultation at which they were clear of disease or date of recurrence, when recurrence was known to have occurred. Of the 2,998 patients remaining, 201 (6.7\%) died as a result of other causes without developing recurrence or new primary, and their follow-up time was censored at their last recorded follow-up visit, and $28(0.9 \%)$ experienced recurrence but had no recorded date of recurrence and were censored at the last follow-up visit when they were free from melanoma.

Clinical characteristics of the 2,998 patients are presented in Table 1; their median length of follow-up, censored at date of recurrence or new primary where applicable, was 2.7 years (range, 0 to 24.7 years). Altogether, 2,036 patients (67.9\%) experienced follow-up of fewer than 5 years because of diagnosis of recurrence or new primary, loss to follow-up, or first primary diagnosis later than December 31, 2004, and 2,553 (85.2\%) experienced follow-up of fewer than 10 years. A substantial proportion of patients (799 of 2,998 patients; $27 \%$ ) were diagnosed within the last 10 years (diagnosed after December 31, 1999), and their follow-up is bounded by the end of the study period (ie, uninformative censoring). For patients diagnosed before 2000 and with no recurrence or new primary, the last recorded follow-up visit was before 10 years of follow-up in $68 \%$ to $75 \%$ of patients, depending on stage.

\section{Recurrence and New Primary Over Time}

Kaplan-Meier curves for time to recurrence stratified by AJCC substage show higher recurrence rates in the first 5 years of follow-up (particularly first year) and lower rates in the subsequent 5 years (Fig 1). New primary melanoma rates within each age group seemed to be fairly constant throughout the 10-year period (Appendix Fig A1, online only).

\section{Prognostic Model}

Two patients who had missing covariate information were excluded from the Cox model, leaving 2,996 patients. For time to recurrence, there was evidence of violation of the proportional 


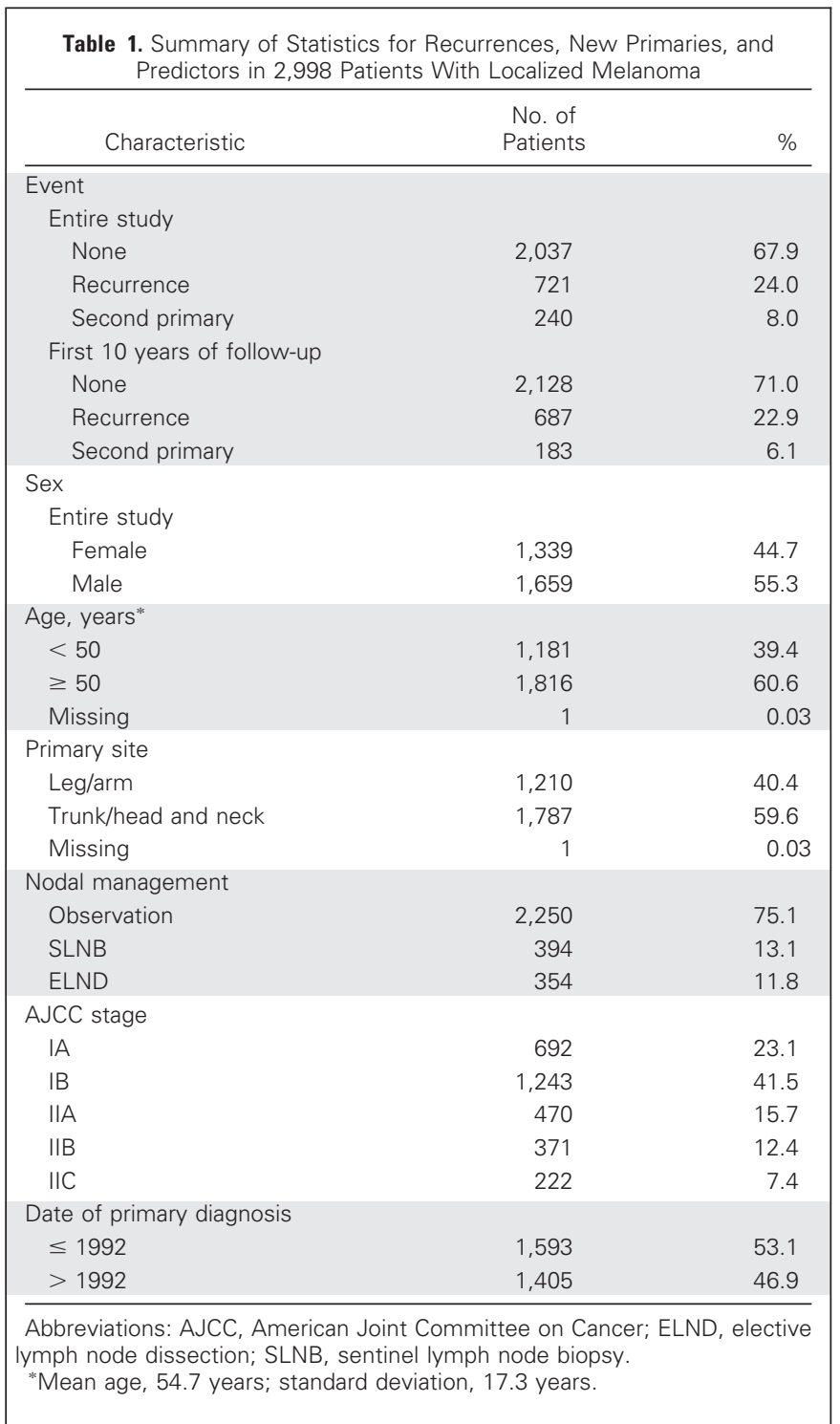

hazards assumption using the whole 10-year follow-up period. Therefore, we built separate models for three periods of follow-up: recurrence within first year of follow-up $(P=0.78$ for test of proportional hazards assumption), within 1 to 5 years $(P=.28)$, and within 5 to 10 years $(P=.96)$. Within the first year, active management of regional lymph nodes protected against early recurrence, whereas increasing AJCC stage predicted increasing risk of early recurrence $(P<.001$ for both; Table 2$)$. Risk was especially high for patients with stages IIB and IIC disease. There was also evidence of modestly increased risk of recurrence for individuals with primary located on the trunk, head, or neck (hazard ratio [HR], $1.45 ; P=.016)$. In the period of 1 to 5 years, nodal management and AJCC stage remained significantly predictive of recurrence, but HRs became less extreme. In the period of 5 to 10 years, only AJCC stage remained predictive of recurrence $(P=.006)$.

For time to new primary, there was no evidence of violation of the proportional hazards assumption $(P=.97)$. Significant predictors were age $(\mathrm{HR}, 1.29 ; P<.001)$ and date of primary diagnosis after 1992

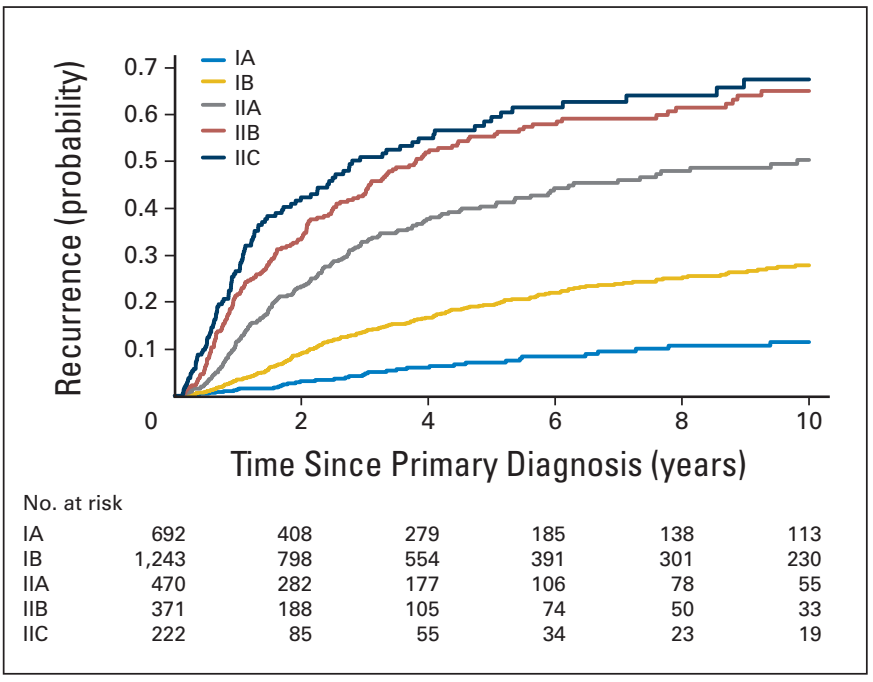

Fig 1. Kaplan-Meier curves showing time to recurrence for localized melanoma according to American Joint Committee on Cancer stage (2009 criteria).

(HR, 2.21; $P<.001$; Table 3 ). AJCC stage was not strongly associated with time to new primary $(P=.053$; Appendix Fig A2, online only). Results of models using a composite outcome of recurrence or new primary are listed in Appendix Table A1 (online only).

The sensitivity analysis using competing risks models took into account the impact of deaths resulting from causes other than melanoma, new primaries as a competing risk for recurrence, and recurrence as a competing risk for new primary. Using these models did not substantially alter the HRs or conclusions.

\section{Modeled Delay in Diagnosis by Monitoring Schedule}

For every 1,000 patients with stage I or II disease beginning follow-up, 229.2 were observed to develop recurrent melanoma and 61.0 to develop new primary within 10 years. Assuming no unscheduled visits prompted by the patient (or family member) detecting an abnormality, more patients experienced a delay in diagnosis of recurrence (Table 4; up to maximum of 1 year) using the less frequent monitoring schedule (schedule two) compared with that currently recommended by Australian and New Zealand guidelines (schedule one), as might be expected, with an estimated 44.9 extra patients per 1,000 experiencing a delay greater than 2 months. More patients also experienced a delay in diagnosis of new primary (Table 5) with schedule two compared with schedule one, but the absolute difference between follow-up schedules was smaller (ie, estimated 9.6 extra patients per 1,000).

If we assume self-detection rates based on published estimates, use of schedule two rather than schedule one results in only a small number of additional patients experiencing a delay in recurrence diagnosis (up to maximum of 3 months; extra 11.3 patients per 1,000 with delay $>2$ months) and even fewer experiencing a delay in new primary diagnosis (up to maximum of 6 months; extra 4.9 per 1,000). The delays in diagnosis of recurrence or new primary considered together and by AJCC substage are presented in the Appendix (online only).

For every 1,000 patients with stage I or II disease who began follow-up, approximately 8,044 monitoring visits would have been needed for schedule one and 5,221 for schedule two. Assuming no loss 
Table 2. Multivariate Cox Proportional Hazard Models for Recurrence Within First 10 Years of Follow-Up in 2,996 Patients With Localized Melanoma

\begin{tabular}{|c|c|c|c|c|c|c|c|c|c|}
\hline \multirow[b]{2}{*}{ Variable } & \multicolumn{3}{|c|}{ First Year } & \multicolumn{3}{|c|}{ Years 1 to $5^{*}$} & \multicolumn{3}{|c|}{ Years 5 to $10 t$} \\
\hline & $\mathrm{HR}$ & $95 \% \mathrm{Cl}$ & $P$ & $\mathrm{HR}$ & $95 \% \mathrm{Cl}$ & $P$ & $\mathrm{HR}$ & $95 \% \mathrm{Cl}$ & $P$ \\
\hline Sex (female $v$ male) & 0.81 & 0.60 to 1.09 & .16 & 0.79 & 0.63 to 0.98 & .03 & 0.69 & 0.42 to 1.11 & .12 \\
\hline Age (per 10-year increase) & 1.07 & 0.98 to 1.17 & .15 & 1.12 & 1.04 to 1.20 & .001 & 1.08 & 0.92 to 1.26 & .36 \\
\hline Primary site (trunk/head and neck $v$ limb) & 1.45 & 1.07 to 1.97 & .016 & 1.07 & 0.86 to 1.33 & .54 & 0.77 & 0.49 to 1.23 & .28 \\
\hline Nodal management & & & $<.001$ & & & $<.001$ & & & .088 \\
\hline Observation & 1 & & & 1 & & & 1 & & \\
\hline SLNB & 0.40 & 0.25 to 0.63 & & 0.50 & 0.35 to 0.71 & & 1.60 & 0.73 to 3.53 & \\
\hline ELND & 0.31 & 0.19 to 0.50 & & 0.50 & 0.37 to 0.68 & & 0.58 & 0.30 to 1.11 & \\
\hline AJCC stage & & & $<.001$ & & & $<.001$ & & & .006 \\
\hline $\mid A$ & 1 & & & 1 & & & 1 & & \\
\hline IB & 2.85 & 1.33 to 6.11 & & 3.47 & 2.18 to 5.51 & & 2.21 & 1.02 to 4.76 & \\
\hline$\| A$ & 11.95 & 5.60 to 25.50 & & 8.98 & 5.53 to 14.60 & & 3.93 & 1.57 to 9.85 & \\
\hline IIB & 21.94 & 10.38 to 46.38 & & 11.18 & 6.82 to 18.33 & & 5.06 & 1.96 to 13.02 & \\
\hline IIC & 28.25 & 13.08 to 61.01 & & 12.18 & 7.15 to 20.75 & & 4.94 & 1.58 to 15.42 & \\
\hline $\begin{array}{c}\text { Date of primary diagnosis }(>1992 \mathrm{v} \\
\leq 1992 \text { reference group) }\end{array}$ & 1.05 & 0.77 to 1.43 & .74 & 1.12 & 0.88 to 1.44 & .35 & 0.91 & 0.49 to 1.69 & .76 \\
\hline
\end{tabular}

Abbreviations: AJCC, American Joint Committee on Cancer; ELND, elective lymph node dissection; HR, hazard ratio; SLNB, sentinel lymph node biopsy.

*Estimates for follow-up years 1 to 5 are for 2,240 patients.

tEstimates for follow-up years 5 to 10 are for 961 patients.

to follow-up and that patients attended all visits until diagnosis or 10 years, then 19,546 monitoring visits would have been needed for schedule one and 12,303 for schedule two.

\section{DISCUSSION}

We estimated that a larger proportion of patients would experience some delay in the diagnosis of recurrence or new primary with the less frequent monitoring strategy (schedule two) compared with that currently recommended by Australian and New Zealand guidelines (schedule one). However, the differences between the two strategies were not large, particularly when we assumed patient

Table 3. Multivariate Cox Proportional Hazards Model for New Primary Within First 10 Years of Follow-Up in 2,996 Patients With Localized Melanoma

\begin{tabular}{llcc}
\multicolumn{1}{c}{ Variable } & HR & $95 \%$ Cl & $P$ \\
\hline Sex (female $v$ male) & 0.80 & 0.58 to 1.10 & .16 \\
Age (per 10-year increase) & 1.29 & 1.17 to 1.44 & $<.001$ \\
$\begin{array}{l}\text { Primary site (trunk/head and } \\
\text { neck } v \text { limb) }\end{array}$ & 1.03 & 0.75 to 1.42 & .85 \\
Nodal management & & & .56 \\
Observation & 1 & & \\
SLNB & 1.17 & 0.77 to 1.77 & \\
ELND & 0.81 & 0.46 to 1.45 & \\
AJCC stage & & & \\
IA & 1 & & \\
IB & 1.12 & 0.77 to 1.63 & \\
IIA & 0.56 & 0.32 to 0.99 & \\
IIB & 0.70 & 0.39 to 1.24 & \\
IIC & 0.95 & 0.50 to 1.78 & \\
Date of primary diagnosis $(>1992 v$ & & & \\
$\quad \leq 1992$ reference group) & 2.21 & 1.55 to 3.16 & $<.001$ \\
\hline
\end{tabular}

Abbreviations: AJCC, American Joint Committee on Cancer; ELND, elective lymph node dissection; HR, hazard ratio; SLNB, sentinel lymph node biopsy. self-detection at previously reported rates of $75 \%$ for recurrence ${ }^{3,7}$ and $50 \%$ for new primary. ${ }^{8}$ It is probable that these differences are small because of the use of AJCC substage to determine follow-up frequency in the novel monitoring strategy (schedule two). Currently, patients with stage IA disease have the same follow-up as patients with stage IB disease, and all stage II patients have the same follow-up regardless of substage, despite clear prognostic differences. On the basis of our modeling, reduction in the number of patients with a delay in diagnosis, without substantially increasing the number of visits, might be possible if monitoring frequency was adjusted according to length of time since treatment, with less frequent follow-up after the first year (ie, monitoring schedule combining features of both schedules one and two).

The novel monitoring schedule required far fewer monitoring visits than are currently recommended. Over 10 years, this would result in approximately 3,000 fewer visits for every 1,000 newly diagnosed patients (ie, approximately three fewer visits per patient over 10 years), assuming dropout rates similar to those observed in this data set, and more than 7,000 fewer visits for every 1,000 newly diagnosed patients (average of seven fewer visits per patient over 10 years), if we assume no loss to follow-up. Not only would these fewer monitoring visits reduce the burden on patients in terms of time and expense in attendance and possible unnecessary anxiety, but they would also represent substantial savings for the health care system.

As might be expected, we found that the risk of recurrence was greatest in the first year of follow-up, with nodal management and AJCC substage strongly predictive of recurrence during this time. In contrast, risk of new primary tended to be constant throughout the 10 -year period, with age and date of primary diagnosis strongly predictive. The finding that risk of new primary was greater for melanomas diagnosed after 1992 was unexpected. It may be explained in part by an increased incidence of melanoma in more recent years ${ }^{12}$ or a shorter time to diagnosis (after 1992, a higher proportion of second primaries were melanoma in situ; data not shown). Age was only 


\begin{tabular}{|c|c|c|c|c|c|c|c|c|}
\hline \multirow{3}{*}{$\begin{array}{l}\text { Delay Threshold } \\
\text { (months) }\end{array}$} & \multicolumn{4}{|c|}{ Scheduled Visits Only* } & \multicolumn{4}{|c|}{ Scheduled and Unscheduled Visits $†$} \\
\hline & \multicolumn{2}{|c|}{ Schedule 1} & \multicolumn{2}{|c|}{ Schedule 2} & \multicolumn{2}{|c|}{ Schedule 1} & \multicolumn{2}{|c|}{ Schedule 2} \\
\hline & No. & $95 \% \mathrm{Cl}$ & No. & $95 \% \mathrm{Cl}$ & No. & $95 \% \mathrm{Cl}$ & No. & $95 \% \mathrm{Cl}$ \\
\hline$>1$ & 169.8 & 160.1 to 179.5 & 192.8 & 183.5 to 201.8 & 42.5 & 38.7 to 46.0 & 48.3 & 45.0 to 51.4 \\
\hline$>2$ & 113.5 & 104.1 to 123.1 & 158.4 & 148.4 to 167.8 & 28.4 & 24.0 to 32.7 & 39.7 & 35.4 to 43.7 \\
\hline$>3$ & 52.7 & 45.2 to 60.4 & 127.7 & 118.4 to 137.4 & \multicolumn{2}{|r|}{$N / A$} & \multicolumn{2}{|r|}{ N/A } \\
\hline$>6$ & 16.0 & 11.7 to 20.7 & 65.0 & 57.0 to 73.0 & \multicolumn{2}{|r|}{ N/A } & \multicolumn{2}{|r|}{ N/A } \\
\hline$>9$ & 9.6 & 6.3 to 13.3 & 34.3 & 28.4 to 40.7 & \multicolumn{2}{|r|}{$N / A$} & \multicolumn{2}{|r|}{ N/A } \\
\hline$>12$ & \multicolumn{2}{|c|}{ N/A } & \multicolumn{2}{|c|}{ N/A } & \multicolumn{2}{|c|}{ N/A } & \multicolumn{2}{|r|}{ N/A } \\
\hline
\end{tabular}

Abbreviation: N/A, not applicable (zero according to either follow-up schedule used or assumption about self-detection).

*Assumes no unscheduled visits because of self-detection.

†Assumes unscheduled visits in $75 \%$ of patients because of self-detection with no delay in diagnosis and maximum delay in diagnosis of 3 months for other patients before scheduled visit or self-detection.

predictive of recurrence 1 to 5 years after diagnosis. However, AJCC stage was still by far the dominant predictor of recurrence, so there would be little added benefit in developing age-specific followup strategies.

Nodal management at MIA changed with the introduction of SLNB in 1992. A significant difference in time to recurrence was found for patients diagnosed before and during 1992 compared with those diagnosed after 1992. This difference with time was no longer significant when nodal management was included in the model. Clinically staged patients are more at risk for developing regional lymph node recurrence and are therefore potentially more likely to experience a delay in diagnosis. A reduction in follow-up frequency might be safe for pathologically staged patients but not as safe for (higher risk) clinically staged patients. However, because nodal staging by SLNB protects against early recurrence, and many more patients now undergo SLNB, this should not affect our conclusions.

Limitations of the study include uncertainty as to actual time of recurrence or new primary melanoma development for some patients in the data set, especially those whose diagnosis was not made until they attended a scheduled follow-up visit. However, monitoring schedules were usually more frequent in the study timeframe than they are now, which, together with the high frequency of self-detection, means we can expect the recorded date of diagnosis to be reasonably accurate. Sensitivity analysis of the self-detection rate showed that the number of patients with a delay in diagnosis of recurrence or new primary decreased almost linearly as the self-detection rate increased. Patients and clinicians may have other objectives for follow-up visits in addition to detection of recurrent or new melanoma ${ }^{13}$; for example, patients want reassurance and the opportunity to ask questions. ${ }^{14}$ These objectives may be more effectively met by fewer visits with a more focused approach to addressing these needs. We have only evaluated 10 years of follow-up after initial treatment and are therefore unable to extrapolate how monitoring strategies would perform beyond this. Many reports have suggested that recurrence tends to plateau at low rates after the first 10 years. ${ }^{9}$ Theoretic modeling does not account for patient adherence to the monitoring schedule; indeed, it is unclear whether adherence to follow-up would increase or decrease if the number of follow-up visits were reduced, an issue that requires more empirical study. Finally, there are likely to be a small number of true stage III patients misclassified as stage II when observation is the nodal management strategy, but this is unlikely to affect the overall conclusions, and if anything, the results presented would be biased toward showing greater benefit from a more frequent monitoring schedule, because such patients are more likely to develop recurrence.

Our study does not address any difference in the ultimate benefit of monitoring, assessed, for example, by incremental loss in life years for each monitoring visit foregone. To do this would require a suitably powered randomized controlled trial.

Table 5. Modeled No. of Patients With Delay in New Primary Diagnosis per 1,000 Patients During 10 Years of Follow-Up

\begin{tabular}{|c|c|c|c|c|c|c|c|c|}
\hline \multirow{3}{*}{$\begin{array}{l}\text { Delay Threshold } \\
\text { (months) }\end{array}$} & \multicolumn{4}{|c|}{ Only Scheduled Visits* } & \multicolumn{4}{|c|}{ Scheduled and Unscheduled Visitst } \\
\hline & \multicolumn{2}{|c|}{ Schedule 1} & \multicolumn{2}{|c|}{ Schedule 2} & \multicolumn{2}{|c|}{ Schedule 1} & \multicolumn{2}{|c|}{ Schedule 2} \\
\hline & No. & $95 \% \mathrm{Cl}$ & No. & $95 \% \mathrm{Cl}$ & No. & $95 \% \mathrm{Cl}$ & No. & $95 \% \mathrm{Cl}$ \\
\hline$>1$ & 44.1 & 37.4 to 51.0 & 47.4 & 40.4 to 54.7 & 22.1 & 19.3 to 24.7 & 23.8 & 21.0 to 26.4 \\
\hline$>2$ & 34.1 & 28.0 to 40.4 & 43.7 & 37.0 to 50.7 & 17.1 & 14.0 to 20.3 & 22.0 & 19.0 to 24.7 \\
\hline$>3$ & 26.1 & 20.7 to 31.7 & 39.1 & 32.7 to 45.7 & 13.1 & 10.0 to 16.3 & 19.6 & 16.7 to 22.7 \\
\hline$>6$ & 6.7 & 4.0 to 9.7 & 23.0 & 17.8 to 28.4 & \multicolumn{2}{|c|}{ N/A } & \multicolumn{2}{|c|}{ N/A } \\
\hline$>9$ & 3.3 & 1.3 to 5.7 & 11.7 & 8.0 to 15.7 & \multicolumn{2}{|c|}{ N/A } & \multicolumn{2}{|c|}{ N/A } \\
\hline$>12$ & \multicolumn{2}{|c|}{ N/A } & \multicolumn{2}{|c|}{$N / A$} & \multicolumn{2}{|c|}{$\mathrm{N} / \mathrm{A}$} & \multicolumn{2}{|c|}{ N/A } \\
\hline
\end{tabular}

Abbreviation: N/A, not applicable (zero according to either follow-up schedule used or assumption about self-detection).

*Assumes no unscheduled visits because of self-detection.

tAssumes unscheduled visits in $50 \%$ of patients because of self-detection with no delay in diagnosis and maximum delay in diagnosis of 6 months for other patients before scheduled visit or self detection. 
Strengths of this study include the large number of patients included with a spectrum of disease likely to be representative of a clinical population of patients treated for localized cutaneous melanoma. Included patients all had detailed pathology results, and we were able to accurately assign AJCC substaging according to the most recent classification published in 2009. We used rigorous statistical methods for the prognostic model and for estimating the delay in diagnosis for different monitoring strategies.

In summary, current guidelines on the frequency of follow-up after treatment for localized melanoma probably provide rather small gains (in terms of earlier diagnosis of recurrence or new primary) at the expense of a large number of additional clinic visits. A less frequent monitoring schedule, such as that proposed in a former MIA publication $^{9}$ and further investigated in the MELFO study, ${ }^{4}$ may decrease unnecessary visits substantially, with only a small consequent delay in the detection of recurrent or new primary melanoma.

\section{AUTHORS' DISCLOSURES OF POTENTIAL CONFLICTS} OF INTEREST

The author(s) indicated no potential conflicts of interest.

\section{AUTHOR CONTRIBUTIONS}

Conception and design: Robin M. Turner, Katy J.L. Bell, Andrew Hayen, Anne Brecht Francken, Bruce Armstrong, John F. Thompson, Les Irwig Provision of study materials or patients: John F. Thompson

Collection and assembly of data: Robin M. Turner, John F. Thompson Data analysis and interpretation: Robin M. Turner, Katy J.L. Bell, Rachael L. Morton, Andrew Hayen, Kirsten Howard, Bruce Armstrong, John F. Thompson

Manuscript writing: All authors

Final approval of manuscript: All authors

\section{REFERENCES}

1. Leiter U, Marghoob AA, Lasithiotakis $K$, et al: Costs of the detection of metastases and follow-up examinations in cutaneous melanoma. Melanoma Res 19:50-57, 2009

2. Altekruse SF, Kosary CL, Krapcho M, et al (eds): SEER Cancer Statistics Review, 1975-2007. National Cancer Institute, Bethesda, MD, 2010 http://seer.cancer.gov/csr/1975_2007

3. Brada M: Is there a need to follow-up cancer patients? Eur J Cancer 31A:655-657, 1995

4. Collins RF, Bekker HL, Dodwell DJ, et al: Follow-up care of patients treated for breast cancer: A structured review. Cancer Treat Rev 30:19-35, 2004

5. Australian National Health and Medical Research Council: Clinical Practice Guidelines for the Management of Melanoma in Australia and
New Zealand. http://www.nhmrc.gov.au/publications/ synopses/cp111syn.htm

6. Dutch Working Group on Melanoma: Skin melanoma (summary) nation-wide guideline, version 1.0. Association of Comprehensive Cancer Centres, Utrecht, the Netherlands, 2006

7. Francken AB, Shaw HM, Accortt NA, et al: Detection of first relapse in cutaneous melanoma patients: Implications for the formulation of evidence-based follow-up guidelines. Ann Surg Oncol 14:1924-1933, 2007

8. Francken AB, Shaw HM, Thompson JF: Detection of second primary cutaneous melanomas. Eur J Surg Oncol 34:587-592, 2008

9. Francken $A B, A c c o r t t N A$, Shaw $H M$, et al: Follow-up schedules after treatment for malignant melanoma. Br J Surg 95:1401-1407, 2008

10. A prospective randomized trial for the evaluation of a theoretical follow-up schedule in cutaneous melanoma patients, the MELFO study (ClinicalTrials gov identifier NCT01018004. http://clinicaltrials.gov/ ct2/show/NCT01018004

11. Montgomery DA, Krupa K, Cooke TG: Alternative methods of follow up in breast cancer: $A$ systematic review of the literature. $\mathrm{Br} J$ Cancer 96:1625-1632, 2007

12. Kokko R, Hakama M, Holli K: Follow-up cost of breast cancer patients with localized disease after primary treatment: A randomized trial. Breast Cancer Res Treat 93:255-260, 2005

13. Walsh $\mathrm{GL}$, O'Connor $\mathrm{M}$, Willis $\mathrm{KM}$, et al: Is follow-up of lung cancer patients after resection medically indicated and cost-effective? Ann Thorac Surg 60:1563-1570, 1995

14. Dancey A, Rayatt S, Courthold J, et al: Views of UK melanoma patients on routine follow-up care. Br J Plast Surg 58:245-250, 2005 